\title{
Factors associated with increased irisin levels in type 1 diabetes mellitus
}

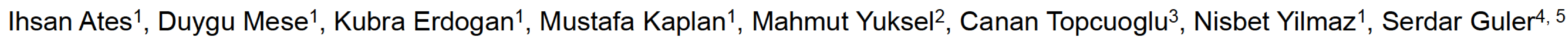

${ }^{1}$ Ankara Numune Training and Research Hospital, Department of Internal Medicine, Ankara, Turkey

2Turkey Yuksek Ihtisas Training and Research Hospital, Department of Gastroenterology, Ankara, Turkey

${ }^{3}$ Ankara Numune Training and Research Hospital, Department of Biochemistry, Ankara, Turkey

${ }^{4}$ Ankara Numune Training and Research Hospital, Department of Biochemistry, Ankara, Turkey

${ }^{5}$ Hitit University School of Medicine, Department of Endocrinology and Metabolism, Çorum, Turkey

\section{Objectives:}

Irisin is a myokine synthesized based on PGC-1a, we think that it is also associated with inflammation Because it is known that PGC-1 $\alpha$ by increasing the synthesis of proinflammatory cytokines, increases the systemic inflammation at the same time. We aimed to determine the irisin level in patients with type 1 diabetes mellitus (T1DM) and to examine the relation of irisin level with inflammation and autoimmunity.

\section{Methods:}

This study included 35 cases diagnosed with T1DM and 36 healthy volunteers. Anti-glutamic acid decarboxylase (Anti-GAD), islet cell antibody (ICA), insulin autoantibody levels of the patients have been measured at the time they were included in the study and were recorded from the patient files. Serum irisin levels were measured by ELISA kit.

\section{Results:}

The median irisin level was determined higher in T1DM group compared to the control group (6.8 $\mathrm{ng} /$ $\mathrm{ml}$ vs. $4.8 \mathrm{ng} / \mathrm{mL}, p=0.022$; respectively). Median irisin level was found higher in anti-GAD ( $p=$ $0.022)$ and ICA $(p=0.044)$ positive groups compared to negative groups. In T1DM group irisin level displayed a positive correlation with glycosylated hemoglobin $(\mathrm{HbA} 1 \mathrm{c})(r=0.377, p<0.001)$ and anti$\operatorname{GAD}(r=0.392, p=0.020)$ and a negative correlation with creatinine $(r=-0390, p=0.021)$. In multivariate regression model, $\mathrm{HbA} 1 \mathrm{c}(\mathrm{B} \pm \mathrm{SE}: 2.76 \pm 17683, p<0.001)$, and anti-GAD (B $\pm \mathrm{SE}: 2.311$ $\pm 0.610, p=0.001$ ) were determined as independent predictors for predicting the irisin level.

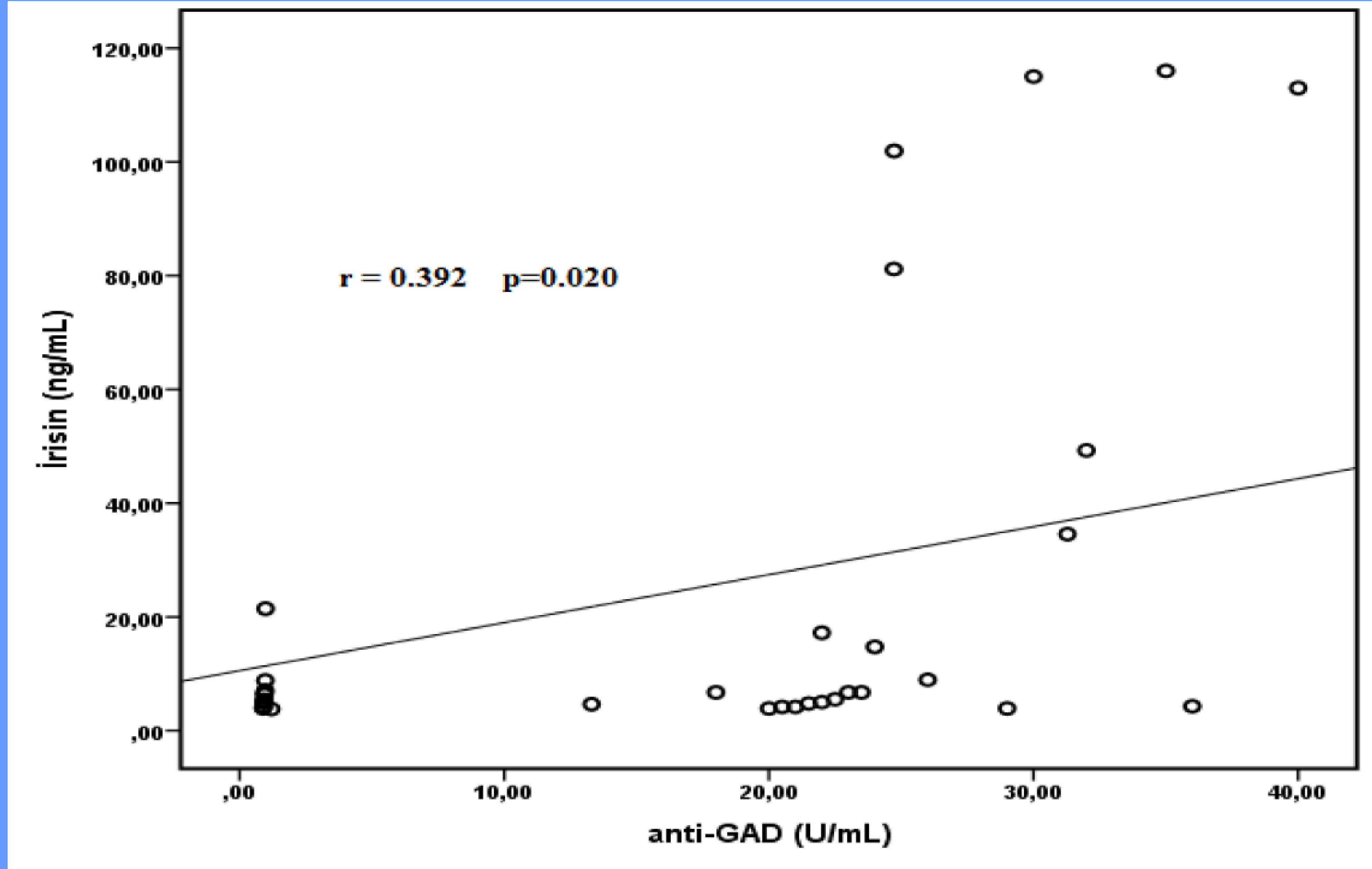

Figure 1: Correlation analysis between the irisin and anti-GAD

Conclusions:

\section{References:}

In patients of T1DM, which chronic inflammation and autoimmunity take part in its etiopathogenesis, anti-GAD level was an independent risk factor for irisin. This suggests that factors such as inflammation and autoimmunity can be effective in the synthesis of irisin.

1. Bostrom P, Wu J, Jedrychowski MP, et al. A PGC1-alpha-dependent myokine that drives brown-fat-like development of white fat and thermogenesis. Nature. 2012; 481(7382): 463-8.

2. Aydin S. Three new players in energy regulation: preptin, adropin and irisin. Peptides. 2014; 56: $94-110$.

3. Ates I, Altay M, Topcuoglu C, Yilmaz FM. Circulating levels of irisin is elevated in hypothyroidism, a case-control study. Arch Endocrinol Metab. 2015: 0. 